\title{
Ocriplasmin for the Treatment of Symptomatic Vitreomacular Adhesion/Traction
}

\author{
Baruch D Kuppermann, MD, PhD
}

Professor of Ophthalmology and Biomedical Engineering; Chief, Retina Service; Vice-Chair, Clinical Research, Ophthalmology School of Medicine, Gavin Herbert Eye Institute, University of California, Irvine, California, US

\begin{abstract}
Ocriplasmin has recently been introduced as a new treatment option for patients with symptomatic vitreomacular adhesion/vitreomacular traction (VMA/VMT). Understanding its potential as well as its limitations is crucial as it becomes an additional tool in the management of these diseases. In this article the overall efficacy and safety of ocriplasmin are reviewed, focusing on the results from the phase III clinical trials as well as recently published case reports and postmarketing data analysis. Efficacy data from ocriplasmin use in a clinical setting support the subanalysis of the phase III clinical trial data. This analysis demonstrated that certain baseline ocular characteristics, namely focal VMA, and absence of epiretinal membrane, are predictive of VMA resolution. Safety findings show that the overall percentage of patients experiencing adverse events during the clinical trial program was low in ocriplasmin-treated patients. Postmarketing surveillance data corroborate findings from the phase III trials, and provide additional insights into the characterization of the safety profile of this new treatment option.
\end{abstract}

\section{Keywords}

Vitreomacular traction, vitreomacular adhesion, ocriplasmin, posterior vitreous detachment, full-thickness macular hole, vitrectomy

Disclosure: Baruch D Kuppermann, MD, PhD, has received clinical research funding from Alcon, Allegro, Allergan, Genentech, GSK, Neurotech, Ophthotech, Regeneron, and ThromboGenics, and has been a consultant to AcuFocus, Aerpio, Alcon, Alimera, Allegro, Allergan, Ampio, Genentech, Neurotech, Novartis, Ophthotech, Regeneron, SecondSight, Staar Surgical, Teva, and ThromboGenics.

Acknowledgments: Editorial assistance was provided by Meridius Health Communications, Inc. (San Diego, AC, US) and funded by ThromboGenics.

Open Access: This article is published under the Creative Commons Attribution Noncommercial License, which permits any noncommercial use, distribution, adaptation, and reproduction provided the original author(s) and source are given appropriate credit.

Received: January 25, 2015 Accepted: February 27, 2015 Citation: US Ophthalmic Review, 2015;8(1):55-9 DOI: 10.17925/usor.2015.8.1.55

Correspondence: Baruch D Kuppermann, MD, PhD, Professor of Ophthalmology and Biomedical Engineering; Chief, Retina Service; Vice-Chair, Clinical Research, Gavin Herbert Eye Institute, University of California, Irvine, 850 Health Sciences Road, Irvine, CA 92697, US. E: bdkupper@uci.edu

Support: The publication of this article was supported by ThromboGenics.

Recent advancements in imaging technology have allowed a more in-depth understanding of the diseases of the vitreoretinal interface (VRI), and have also changed how we evaluate the effectiveness of different treatment options. Until the development of optical coherence tomography (OCT), no practical method was widely available for visualizing and evaluating diseases of the VRI, and no consensus on the definition and classification of these diseases had been developed.

The development of Ост imaging technology has allowed better visualization of the complex and inevitable set of events that occur as the eye ages. Concurrent liquefaction of the vitreous gel and progressive posterior vitreous cortex separation ultimately lead to, in most eyes, nonpathologic posterior vitreous detachment (PVD). ${ }^{1,2}$ In some cases, however, incomplete VRI separation can result in anomalous PVD with the potential for the development of pathologic features. ${ }^{2,3}$ As defined by the International Vitreomacular Traction Study Group classification system, ${ }^{2}$ anomalous PVD is a partial vitreous detachment with persistent attachment in the macular region, resulting in tractional deformation of retinal tissue. Elevation of the cortical vitreous above the retinal surface, with the vitreous remaining attached within a $3 \mathrm{~mm}$ radius of the fovea, is defined as vitreomacular adhesion (VMA). ${ }^{2}$ Importantly, in the case of VMA, which is a normal part of the aging process in many eyes, the retina displays no change in contour or morphologic features on OCT, and therefore people with VMA generally experience no visual impairment. In some cases, the progression of PVD can lead to periods of excessive traction on the macula and distortion of the retinal architecture, which is then characterized as vitreomacular traction (VMT). Such traction can result in intraretinal pseudocyst formation, elevation of the fovea from the retinal pigment epithelium (RPE), or a combination that can result in reduced or distorted vision. ${ }^{4}$ The presence of pseudocysts frequently 


\section{Table 1: The International Vitreomacular Traction Study Classification System for Vitreomacular Adhesion, Traction, and Macular Hole}

\begin{tabular}{|c|c|}
\hline Classification & Subclassification \\
\hline Vitreomacular adhesion & $\begin{array}{l}\text { Size: focal }(\leq 1,500 \mu \mathrm{m}) \text { or broad }(>1,500 \mu \mathrm{m}) \\
\text { Isolated or concurrent }\end{array}$ \\
\hline Vitreomacular traction & $\begin{array}{l}\text { Size: focal }(\leq 1,500 \mu \mathrm{m}) \text { or broad }(>1,500 \mu \mathrm{m}) \\
\text { Isolated or concurrent }\end{array}$ \\
\hline Full-thickness macular hole & $\begin{array}{l}\text { Size: small }(\leq 250 \mu \mathrm{m}) \text {, medium ( }>250 \text { to } \leq 400 \mu \mathrm{m}) \text {, or } \\
\text { large ( }>400 \mu \mathrm{m}) \\
\text { Status of vitreous: with or without VMT } \\
\text { Cause: primary or secondary }\end{array}$ \\
\hline
\end{tabular}

VMT = vitreomacular traction. Reproduced with permission from Duker et al., 2013. ${ }^{2}$

\section{Table 2: Ocriplasmin Efficacy in a Clinical Setting}

\begin{tabular}{llll} 
Publication & N & VMA Resolution, \% (n/N) & FTMH Closure, \% (n/N) \\
Kim et al. ${ }^{15}$ & 19 & $42(8 / 19)$ & $50(3 / 6)$ \\
\hline Singh et al. ${ }^{17}$ & 17 & $47(8 / 17)$ & $80(4 / 5)$ \\
\hline Knudsen et al. $^{16}$ & 8 & $63(5 / 8)$ & NA \\
\hline
\end{tabular}

FTMH = full-thickness macular hole; NA = not applicable; VMA = vitreomacular adhesion.

is associated with vision impairment, and once traction is released, pseudocysts generally resolve with improvement in vision. ${ }^{5}$

Both VMA and VMT can be subclassified into either focal $(\leq 1,500 \mu \mathrm{m})$ or broad (>1,500 $\mu \mathrm{m})$ adhesions based on attachment size (see Table 1). Focal areas of vitreous attachment with traction tend to distort the foveal surface, whereas broad areas of attachment with traction can cause generalized thickening of the macula, vascular leakage on fluorescein angiography, macular schisis, and cystoid macular edema. ${ }^{2}$

Excessive traction on the inner retina may also result in the development of a full-thickness macular hole (FTMH), defined as an anatomic defect in the fovea featuring interruption of all neural retinal layers from the internal limiting membrane (ILM) to the RPE. ${ }^{2,3}$ FTMH can have either persistent VMT or complete release of the vitreous. Using an OCT-based system, the presence of continued VMT and measurements of minimum hole width (aperture size) become important as the aperture size of the FTMH and the presence of residual VMT can help determine appropriate treatment options. ${ }^{3}$

In a small number of cases, VMT may resolve spontaneously, with multiple factors contributing to the likelihood of spontaneous resolution, including the tenacity of the adhesion between the vitreous gel and macula, and presence of an epiretinal membrane (ERM) ${ }^{4,6-8}$ Consequently, spontaneous resolution is hard to predict, and watchful waiting has been the standard of care for early or mild cases, with pars plana vitrectomy (PPV) usually reserved for more chronic or severe cases. ${ }^{9}$ Until the approval of ocriplasmin in 2012, vitrectomy and watchful waiting were the only management strategies for patients with tractional diseases of the VMI. Therefore, understanding the appropriate clinical use as well as the limitations of ocriplasmin is crucial, as it becomes an additional tool for the treatment of symptomatic VMA/VMT. Financial considerations are definitely a factor in the choice of treatment strategy. The cost of the drug should be weighed against the benefits and needs to be discussed thoroughly with the patients along with the clinical aspects of disease management.

\section{Ocriplasmin Efficacy}

Ocriplasmin is a truncated form of plasmin that enzymatically cleaves structural proteins, including laminin and fibronectin, at the VMI. Results of preclinical and clinical studies have suggested that ocriplasmin can induce vitreous liquefaction and separation from the retina. ${ }^{10-12}$

In its initial phase of development, ocriplasmin was evaluated as an adjunct to vitrectomy to assess its effectiveness in the achievement of complete PVD. A preliminary clinical study assessed intravitreal ocriplasmin at varying doses administered either 1-2 hours, 24 hours, or 7 days before planned PPV for VMT. ${ }^{12}$ Increasing the duration of observation after exposure to ocriplasmin was associated with a progressive increase in the incidence of complete PVD achievement. Subsequently, two phase III clinical trials with similar designs were conducted to assess the efficacy and safety of a single intravitreal injection of ocriplasmin in patients with VMT with or without associated FTMH (400 $\mu \mathrm{m}$ or less)..$^{13}$ The percentage of eyes achieving the primary endpoint, which was resolution of VMA at day 28 , was significantly greater with ocriplasmin than with vehicle: $26.5 \%$ versus $10.1 \%(p<0.001)$, respectively. ${ }^{13}$ In addition, a significantly higher proportion of patients achieved the secondary endpoints compared with those receiving the vehicle, including: (a) $13.4 \%$ versus $3.7 \%(p<0.001)$ of ocriplasmin versus vehicle-treated patients achieving total PVD at day 28; (b) $40.6 \%$ versus $10.6 \%(p<0.001)$ of ocriplasmin versus vehicle-treated patients achieving nonsurgical closure of FTMH at day 28; and (c) $12.3 \%$ versus $6.4 \%$ ( $p<0.02$ ) of ocriplasmin versus vehicle-treated patients achieving $\geq 3$ lines improvement in visual acuity (VA) at month 6.13

A subanalysis of the data from the phase III clinical trials identified patient baseline characteristics that may be associated with VMA resolution after treatment with ocriplasmin. There were five characteristics identified as positive predictors of VMA resolution: age $<65$ years, presence of FTMH at baseline, absence of ERM, VMA diameter $\leq 1,500 \mu \mathrm{m}$, and phakic lens status. ${ }^{14}$

Recent publications have supported the absence of ERM and the presence of focal adhesion $(\leq 1,500 \mu \mathrm{m})$ as positive predictors of VMA resolution after treatment with ocriplasmin. The first case series on ocriplasmin was published by Kim et al., ${ }^{15}$ which reported an overall VMA resolution rate of $42 \%(8 / 19)$. When analyzing VMA resolution rates by subgroups with different baseline characteristics, 2/8 patients (25\%) with ERM versus $5 / 11$ (45.5 \%) without ERM achieved nonsurgical VMA resolution. When considering size of adhesion, none of the cases with VMA diameter $>1,500$ $\mu \mathrm{m}$ had VMA resolution, whereas 8/16 (50 \%) patients with VMA diameter $\leq 1,500 \mu \mathrm{m}$ achieved resolution of VMA. ${ }^{15}$ Additional publications have also confirmed that improved rates of VMA resolution can be obtained with careful patient selection, with VMA resolution rates as high as $63 \%$ (see Table 2). ${ }^{15-17}$

Regarding visual outcomes, recent publications have reported modest gains, and highlight the need for longer-term follow-up studies. Kim et al. reported modest VA gains, with eight out of 19 (41\%) patients treated gaining 2 ETDRS lines or fewer, and another $41 \%$ with no change in baseline vision. ${ }^{15}$ Singh et al. reported that although there was a trend of improved VA in the group of patients who achieved VMA release, this was not statistically different from the patients without VMA release. ${ }^{17}$ The authors also note that the use of standard Snellen acuity, rather than protocol VA, and the short follow-up period might have blunted the VA outcomes. 


\section{Ocriplasmin Safety}

In the pivotal phase III ocriplasmin clinical trials, the proportion of patients who had any ocular adverse event in the study eye was $68.4 \%$ in the ocriplasmin group and $53.5 \%$ in the vehicle group $(p<0.001$ ) (see Table 3). Interestingly, this difference was driven primarily by adverse events known to be associated with vitreous detachment, as the most common ocular adverse event in the study eye was vitreous floaters, reported by $16.8 \%$ of patients in the ocriplasmin group and $7.5 \%$ of those in the vehicle group. ${ }^{13}$ Retinal tears or detachments were diagnosed in $1.9 \%$ of the patients given ocriplasmin compared with $4.3 \%$ of those given vehicle. Most retinal breaks in both groups occurred during or after vitrectomy; two $(0.4 \%)$ retinal detachments in the ocriplasmin group and one $(0.5 \%)$ retinal tear in the vehicle group occurred prior to any vitrectomy.

Most suspected treatment-related ocular adverse events were not serious, mild in severity, and occurred within 7 days after injection. Of the 465 patients treated in the phase III studies, 36 patients $(7.7 \%)$ reported an acute reduction in VA. Three patients (0.65\%) experienced a transient significant $(\leq 20 / 200)$ reduction in VA. Time-to-onset was less than 7 days, and median time to resolution was 14 days. There were 36 patients with acute vision loss, and $83 \%$ resolved by the 6-month study endpoint. Vision loss persisted in six patients ( $1.3 \%$ of total patients): five of these six patients had complications related to vitrectomy $(n=4)$ or needed vitrectomy $(n=1)$; one of the six patients had vision loss for unknown reasons despite VMA resolution and anatomic improvement. Overall, most cases were transient, as by the end of the 6-month study reduced VA was reported in approximately $1 \%$ of patients in both the ocriplasmin- and vehicle-treated groups (see Figure 1). ${ }^{13}$

In addition, there were a total of 18 patients from additional completed studies who experienced dyschromatopsia and/or electroretinogram (ERG) changes. Dyschromatopsia (generally described as yellowish vision) was reported in 16 patients. The majority of cases were reported from two uncontrolled open-label clinical studies (TG-MV-008 and TGMV-010). Median time to onset was 1 day, and median time to resolution was 3 months. In 13 of the 16 cases the dyschromatopsia resolved. ERG changes (a- and b-wave amplitude decrease) were reported in 10 patients. Similar to the dyschromatopsia cases, the majority of cases were reported from the same two uncontrolled open-label clinical studies. The median time to onset was 1 week, and median time to resolution was 6 months, with the majority of the patients showing a trend to recovery of the wave amplitude towards baseline and VA returning to baseline or better.

Case series reports have also contributed to a better characterization of the safety profile of ocriplasmin. A recent case series reported changes in the ellipsoid zone following treatment with ocriplasmin, and the authors found that the rate of ellipsoid zone loss was higher in patients with resolution of VMT (75 \% in responders versus $11 \%$ in nonresponders). ${ }^{17}$ The case series also demonstrated that of 17 patients treated, seven (41\%) experienced ellipsoid zone loss on OCT, and this was transient in all patients with a mean time to resolution of 29 days. ${ }^{17}$

Two additional cases have been published last year, describing the overall clinical course of two patients treated with ocriplasmin. Fahim et al. ${ }^{18}$ reported a single case study of a 63-year-old female with VMA and $\mathrm{MH}$ followed up to 9 days. At 9 days, the patient suffered from acute visual
Table 3: Summary of Safety Results from the Phase III Clinical Trials

\begin{tabular}{lll} 
Preferred Term, $\mathrm{n}(\%)$ & Vehicle $(\mathrm{n}=187)$ & Ocriplasmin $(\mathrm{n}=465)$ \\
\hline Any ocular adverse event & $100(53.5)$ & $318(68.4)$ \\
\hline Vitreous floaters & $14(7.5)$ & $78(16.8)$ \\
\hline Photopsias & $5(2.7)$ & $55(11.8)$ \\
\hline Conjunctival hemorrhage & $24(12.8)$ & $68(14.6)$ \\
\hline Injection-related eye pain & $11(5.9)$ & $63(13.5)$ \\
\hline Blurred vision & $6(3.2)$ & $40(8.6)$ \\
\hline Visual impairment & $3(1.6)$ & $25(5.4)$ \\
\hline Increased intraocular pressure & $10(5.3)$ & $18(3.9)$ \\
\hline Retinal tear & $5(2.7)$ & $6(1.3)$ \\
\hline Cataract & $17(9.1)$ & $26(5.6)$ \\
Any ocular serious adverse event & $20(10.7)$ & $36(7.7)$ \\
\hline Macular hole & $16(8.6)$ & $24(5.2)$ \\
\hline Retinal detachment & $3(1.6)$ & $2(0.4)$ \\
\hline Reduced visual acuity & $1(0.5)$ & $3(0.6)$ \\
\hline
\end{tabular}

Adapted from Stalmans et al., 2012. ${ }^{3}$

\section{Figure 1: Acute Reduction in Visual Acuity Over Time}

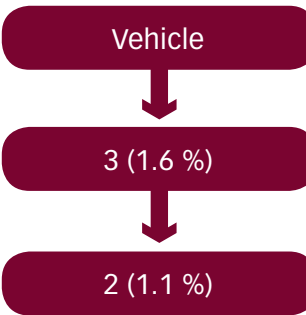

Day 7

Ocriplasmin

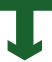

$36(7.7 \%)$

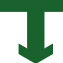

Month 6

$6(1.3 \%)$

loss following ocriplasmin injection. PVD was present, with a FTMH noted and OCT and ERG abnormalities observed. The authors concluded that long-term follow-up of this patient, as well as other patients treated with ocriplasmin, is required. Tibbetts et al. ${ }^{19}$ reported the case of a 71-yearold female with VMT that successfully resolved following treatment with ocriplasmin. The patient was reported to have acute VA decrease from $20 / 60$ to 20/200 at day 1 , followed by improvement to 20/40 at month 4 . Disruption of the ellipsoid zone on OCT was also noted at day 1 , with improvement reported by 2 months. In this patient, ERG abnormalities were observed, with rods (scotopic vision) affected more than cones (photopic vision); these abnormalities persisted to 4 months. The authors conclude that further work is necessary to identify potential effects of ocriplasmin on photoreceptors, and to identify those patients who may be at risk of these adverse events.

Periodic reporting plays a major role in the safety assessment of a drug, as spontaneous reporting allows health authorities to gather safety information on newly available treatment options. Considered one of the most important aggregate reports worldwide, the Periodic Benefit-Risk Evaluation Report (PBRER) is submitted to drug regulatory authorities in Europe, the US, and Japan, as well as in other countries. ${ }^{20}$ It is updated biannually and contains the results from an ongoing collection of postmarketing surveillance data. The focus of the PBRER is on the benefit-risk profile of the drug, which includes a review of relevant safety information compiled for a drug product in market and throughout its development. The safety reports consist of preclinical, clinical trial, and 


\section{Table 4: Comparison of Frequency of Adverse Events in Clinical Trial Program and Postmarketing Period Ending 4/16/2014}

\begin{tabular}{lll}
$\begin{array}{l}\text { Adverse Events of Special } \\
\text { Interest }\end{array}$ & $\begin{array}{l}\text { Clinical Trial Program } \\
\text { Frequency (\%) } \\
\mathrm{N}=1,115\end{array}$ & $\begin{array}{l}\text { Postmarketing } \\
\text { Frequency (\%) } \\
\mathrm{N}=10,770^{+}\end{array}$ \\
$\begin{array}{l}\text { Serious/severe reductions in } \\
\text { visual acuity }\end{array}$ & 0.9 & 1.3 \\
$\begin{array}{l}\text { ERG changes } \\
\text { 10 cases reported in } \\
\text { BLA submission }\end{array}$ & 9 cases reported \\
\hline Dyschromatopsia & 1.7 & 0.5 \\
\hline Retinal tear/detachment & $0.4^{*+1}$ & 0.5 \\
\hline $\begin{array}{l}\text { Lens subluxation/phacodonesis } \\
\text { Impaired pupillary reflex }\end{array}$ & 2 cases* & 0.1 \\
\hline Ellipsoid zone changes** & NA & 0.2 \\
Retinal vessel findings & 1 case reported & 0.3 \\
New or worsening macular hole & $6.7^{*}$ & 0.1 \\
\hline
\end{tabular}

${ }^{+}$Estimated from the number of doses distributed; ${ }^{+}$Includes previtrectomy occurrences only; *Includes data from phase III clinical trials only $(n=465) ; * *$ Identified by medical review and comprise the following Medical Dictionary for Regulatory Activities (MedDRA) terms: retinal toxicity, retinal injury, retinal degeneration, macular degeneration, detachment of retinal pigment epithelium, ophthalmologic examination abnormal. BLA = Biologics License Application; ERG = electroretinogram. Data on file. ThromboGenics, Inc. 2013. Ocriplasmin 3rd Periodic Benefit-Risk Evaluation Report. ThromboGenics NV. June 23, 2014.

postmarketing data, which are primarily based on voluntary reports by retina specialists and other healthcare professionals. Although the realworld experiences extend beyond the limited number of a clinical trial and contribute to a better characterization of the safety profile of a drug, there may be underreporting due to the nature of voluntary reporting.

For the last reporting period available (PBRER 3), the estimated cumulative exposure to ocriplasmin in completed and ongoing trials was 1,115 eyes that received ocriplasmin at any dose. For postmarketing exposure data, the number of exposures was estimated from the number of doses distributed as of April 16, 2014-the date of last reporting—which was 10,770.

Table 4 summarizes the frequency of adverse events of special interest for both the clinical trial program as well as for the postmarketing period. Significant reduction in VA was observed in $0.9 \%$ of ocriplasmin subjects in the clinical trials program. From the postmarketing analysis, reduction in VA was reported with a frequency of $1.3 \%$. Dyschromatopsia was observed in $1.7 \%$ of subjects in the clinical trials program and had a frequency of $0.5 \%$ in the postmarketing experience. There were two cases of lens subluxation/phacodonesis reported in the clinical trial program, and seven cases $(0.1 \%)$ reported in the postmarketing period. Inner segment/outer segment (IS/OS) junction (ellipsoid zone) findings, which were reported only during the postmarketing period, had a reported frequency of $0.3 \%$.

Overall, the recently published case reports add to the existing body of evidence supporting ocriplasmin. There is no suggestion that the risk-benefit profile of ocriplasmin, as demonstrated in the Microplasmin for Intravitreous Injection-Traction Re-lease Without Surgical Treatment (MIVI-TRUST) studies involving more than 600 patients, is altered by these case studies. In addition, the aggregate reports further support the safety profile of ocriplasmin, as despite significant differences in reporting methods, the types of adverse events are mostly consistent between the clinical trials and the postmarketing experience. It should be noted that the reported clinical course post ocriplasmin injection correlate well with the anticipated anatomic changes that occur in pharmacologic vitreolysis. The same or similar symptoms can also be experienced by patients that undergo vitrectomy, a surgical approach with a relatively more prolonged treatment course.

\section{Overall Conclusions}

Ocriplasmin is a novel, efficacious treatment option for patients with VMA, as demonstrated by the results from the phase III trials, ${ }^{13}$ as well as recently published reports. ${ }^{15-17}$ Efficacy data from ocriplasmin use in a clinical setting have supported the subanalysis of phase III data, which demonstrated that certain baseline ocular characteristics, primarily focal VMA, and absence of ERM, are predictive of VMA resolution and are important for optimal patient selection.

Safety findings show that the overall percentage of patients experiencing adverse events during the clinical trial program was low in ocriplasmintreated patients. Ocriplasmin was well-tolerated and most treatmentrelated ocular adverse events were not serious, were mild in severity, and occurred within 7 days post injection. ${ }^{13,21}$ Postmarketing surveillance data corroborate findings from the phase III trials, and provide additional insights into the characterization of the safety profile of ocriplasmin. Further understanding is needed on some of the observations that had not been reported in the phase III trials, such as ellipsoid zone changes. Identifying which patients are more likely to develop particular adverse events is important; however, as of yet the adverse events appear to be unpredictable. Interestingly, ellipsoid zone changes may be a positive predictor of response, as it was reported to occur more frequently in patients who had VMA release. ${ }^{17}$ Further studies are needed to clarify the mechanisms involved.

A number of phase IIIb/IV studies are currently ongoing to address some of these questions, and to further characterize the long-term safety profile of ocriplasmin. The Ocriplasmin for Treatment for Symptomatic Vitreomacular Adhesion including Macular Hole (OASIS) study will generate long-term data following treatment with ocriplasmin. This masked study is designed to assess anatomical and functional outcomes following a single intravitreal injection of ocriplasmin $0.125 \mathrm{mg}$ in subjects with symptomatic VMA/VMT including $\mathrm{MH}$, and includes full-field ERGs in a substudy of 62 patients. Patients in the study are being followed up for a 24-month period post injection. In addition, the Ocriplasmin Research to Better Inform Treatment (ORBIT) study was initiated in March of 2014 and aims to recruit 1,500 patients with symptomatic VMA/VMT across 120 retina centers in the US. This prospective, observational study will assess a number of parameters including resolution of VMA, FTMH closure, changes in VA, and occurrence and time to vitrectomy. It will also monitor adverse drug reactions (ADRs) and changes from baseline in ocular signs and symptoms, such as metamorphopsia, over time.

Two additional studies are also enrolling patients in Europe and Canada. The INvestigation of JETREA in Patients With Confirmed Vitreomacular Traction (INJECT) study is a noninterventional, multicenter, prospective study in VMT patients treated with ocriplasmin. Enrolled patients are followed for 12 months, and frequency and timing of postinjection visits are at the discretion of the treating physician. The Assessment of Anatomical 
and Functional Outcomes in the Ocriplasmin for Vitreomacular Traction (VMT) Intravitreal Injection Decisions (OVIID 1) trial is a multicenter, prospective, single-arm study in which patients diagnosed with VMT/ SVMA are treated with ocriplasmin $125 \mu \mathrm{g}$ by intravitreal injection as per the country's product label. The study includes a total of six planned visits and safety is assessed through the use of reported adverse events and ophthalmologic examinations. Results from these studies will provide insights into presenting characteristics of patients with VMT and VMT with $\mathrm{MH}$, who receive ocriplasmin in a real-world setting, and will contribute to the further characterization of ocriplasmin efficacy, safety profile, and global treatment patterns.

Based on the available data, ocriplasmin can be considered a safe treatment option, especially considering its mechanism of action (enzymatic cleavage of structural proteins) as well as the fact that it is commonly used as an alternative to surgical vitrectomy, which has its own set of side effects. In fact, ocriplasmin could be viewed as a gentler form of vitrectomy, as it pharmacologically induces an increase in traction, accelerating the process that leads to VMA release. In addition, the frequency of certain types of adverse events observed following treatment with ocriplasmin is consistent with what has been reported for vitrectomy. A recently published systematic review of the literature to determine the safety and efficacy of PPV for vitreomacular syndrome identified focal, petechial, spontaneously resolving retinal hemorrhage, and peripheral retinal breaks as the most frequent intraoperative complications, with $6 \%$ and $2 \%$ of eyes affected, respectively. For postoperative complications, the events with the highest percentage of eyes affected were cataract (35\%), cataract surgery (11\%), epimacular/ERM (6\%), and retinal detachment (5\%)..22 The authors also noted that approximately one-third of eyes treated with PPV for VMT gained 2 Snellen lines, and suggested that these gains may be less than anticipated. As additional data on ocriplasmin efficacy is being generated, a closer evaluation of the outcomes and adverse events associated with vitrectomy should also be considered.

Overall, both the clinical trial data and the emerging real world experience show that ocriplasmin appears to be an effective and safe treatment option, and provides a new addition to our armamentarium in the management of vitreo-retinal interface disorders.
1. Sebag J, Classifying posterior vitreous detachment: a new way to look at the invisible, Br J Ophthalmol, 1997:81:521.

2. Duker JS, Kaiser PK, Binder $S$, et al., The International Vitreomacular Traction Study Group classification of vitreomacular adhesion, traction, and macular hole, Ophthalmology, 2013;120:2611-9.

3. Stalmans P, Duker JS, Kaiser PK, et al., OCT-based interpretation of the vitreomacular interface and indications for pharmacologic vitreolysis, Retina, 2013;33:2003-11.

4. Johnson MW, Posterior vitreous detachment: evolution and role Johnson MW, Posterior vitreous detachment. evolutior

5. Haouchine B, Massin P, Gaudric A, Foveal pseudocyst as the first step in macular hole formation: a prospective study by optical coherence tomography, Ophthalmology, 2001;108:15-22

6. Hickichi T, Yoshida A, Trempe CL, Course of vitreomacular traction syndrome, Am J Ophthalmol, 1995;119:55-61.

7. Weinand $F$, Jung A, Becker R, Pavlovic S, Spontaneous resolution of vitreomacular traction syndrome, Ophthalmologe 2009:106:44-6.

8. Odrobina D, Michalewska Z, Michalewski J, et al., Long-term evaluation of vitreomacular traction disorder in spectral-domain optical coherence tomography, Retina, 2011;31:324-31
9. Steel DH, Lotery AJ, Idiopathic vitreomacular traction and macular hole: a comprehensive review of pathophysiology, diagnosis, and treatment, Eye (Lond), 2013:27(Suppl. 1):S1-21.

10. de Smet MD, Valmaggia C, Zarranz-Ventura J, Willekens B, Microplasmin: ex vivo characterization of its activity in porcine vitreous, Invest Ophthalmol Vis Sci, 2009;50:814-9.

11. Gandorfer A, Rohleder M, Sethi C, et al., Posterior vitreous detachment induced by microplasmin, Invest Ophthalmol Vis Sci, 2004;45:641-7.

12. de Smet MD, Gandorfer A, Stalmans P, et al., Microplasmin intravitreal administration in patients with vitreomacular traction scheduled for vitrectomy: the MIVI I trial, Ophthalmology, 2009:116:1349-55.

13. Stalmans P, Benz M, Gandorfer A, et al., Enzymatic vitreolysis with ocriplasmin for vitreomacular traction and macular hole, N Engl J Med, 2012;367:606-15.

14. Ray S, Independent baseline features predictive of pharmacologic VMA resolution in the phase III ocriplasmin clinical program. Presented at AAO Retina Subspecialty Day, Chicago, IL, 2012

15. Kim BT, SchwartZ SG, Smiddy WE, et al., Initial outcomes following intravitreal ocriplasmin for treatment of symptomatic vitreomacular adhesion, Ophthalmic Surg Lasers Imaging Retina
2013:44:334-3

16. Knudsen VM, Kozak I, A retrospective study of a single practice use of ocriplasmin in the treatment of vitreomacular traction, Saudi I Ophthalmol, 2014;28:139-44.

17. Singh RP, Li A, Bedi R, Srivastava S, et al., Anatomical and visual outcomes following ocriplasmin treatment for symptomatic vitreomacular traction syndrome, $\mathrm{Br} J$ Ophthalmol, 2014;98:356-60.

18. Fahim AT, Khan NW, Johnson MW, Acute panretinal structural and functional abnormalities after intravitreous ocriplasmin injection, JAMA Ophthalmol, 2014;132:484-6.

19. Tibbetts $M D$, Reichel $E$, Witkin $A J$, Vision loss after intravitreal ocriplasmin: correlation of spectral-domain optical coherence tomography and electroretinography, JAMA Ophthalmol, 2014;132:487-90

20. Gliklich RE, Dreyer NA (eds), Registries for evaluating patient outcomes: A user's guide, 2nd ed., Rockville, MD: Agency for Healthcare Research and Quality (US), 2010.

21. Kuppermann BD, Ocriplasmin for pharmacologic vitreolysis, Retina, 2012;32(Suppl. 2):S225-8.

22. Jackson TL, Nicod E, Angelis A, et al., Pars plana vitrectomy for vitreomacular traction syndrome: a systematic review and metaanalysis of safety and efficacy, Retina, 2013;33:2012-7. 